\title{
How the mind shapes action: Offline contexts modulate involuntary episodic retrieval
}

\author{
Christian Frings $^{1} \cdot$ Iring Koch $^{2} \cdot$ Birte Moeller $^{1}$
}

Published online: 23 August 2017

(C) The Psychonomic Society, Inc. 2017

\begin{abstract}
Involuntary retrieval of previous stimulus-response episodes is a centerpiece of many theories of priming, episodic binding, and action control. Typically it is assumed that by repeating a stimulus from trial $n-1$ to trial $n$, involuntary retrieval is triggered in a nearly automatic fashion, facilitating (or interfering with) the to-be-executed action. Here we argue that changes in the offline context weaken the involuntary retrieval of previous episodes (the offline context is defined to be the information presented before or after the focal stimulus). In four conditions differing in cue modality and target modality, retrieval was diminished if participants changed the target selection criterion (as indicated by a cue presented before the selection took place) while they still performed the same task. Thus, solely through changes in the offline context (cue or selection criterion), involuntary retrieval can be weakened in an effective way.
\end{abstract}

Keywords Cognitive and attentional control · Task switching $\cdot$ Executive control $\cdot$ Perception and action

Our world is full of contingencies. Stimuli, contexts, tasks, and people are encountered again and again and repeatedly occur together. Cognitive mechanisms have evolved that adapt to these contingencies; a prominent example is the involuntary

Christian Frings

chfrings@uni-trier.de

1 Institute of Psychology, University of Trier, Campus I, Trier, Germany

2 Institute of Psychology, RWTH Aachen University, Aachen, Germany retrieval of previous episodes - that is, even stimuli or contexts to which people do not attend, or even that they try to ignore (e.g., distractor stimuli), can elicit retrieval of previous episodes. As a result, retrieval is not only relevant to the research field of memory but has gained increased interest for models and theories of action control. In fact, involuntary retrieval is the centerpiece of many theories of memory, priming, or action control (e.g., Henson, Eckstein, Waszak, Frings, \& Horner, 2014; Hommel, 1998, 2004; Logan, 1990) that focus on (partially) automatic behavior (e.g., Moors \& De Houwer, 2006). The basic idea is that when one encounters a specific repeated episode, the last memory entry of this episode is automatically retrieved from memory, and the retrieved information modulates behavior (typically by facilitating it). As a result, humans do not have to use component or algorithmic processes all the time (Roediger \& McDermott, 1993), but can rely on "solutions from the past."

Yet, retrieval is not always beneficial and must be guided so as to prevent an overflow of episodes - for example, if one encounters just familiar objects. One well-known variable that modulates retrieval in general is the context; retrieval becomes stronger with increasing similarity of contexts in encounter $n$ and encounter $n-1$. Of course, the "context" in itself is a concept that is difficult to define. One coarse-grained differentiation borrowed from memory research might be to separate online versus offline contexts. The online context is defined to be the information presented simultaneously along with the focal stimulus, whereas the offline context is defined to be the information presented before or after the focal stimulus. For example, cued recall in memory research (e.g., Thomson \& Tulving, 1970) is understood as using cues to assist in retrieving the to-be-recalled items; that is, the cues serve as an online context during retrieval. Yet, online contexts do not have to consist of external information; they can also include everything that is not presented or cannot be 
perceived in the environment, such as intentions, moods, or memorized instructions. For instance, the debate on moodstate-dependent memory (Kenealy, 1997; Ucros, 1989) suggests that performance is best when the mood at retrieval matches the mood at encoding.

In contrast, offline contexts modulate retrieval, although the context is not presented when the retrieval takes place. For instance, in the retrieval-induced forgetting and directed-forgetting paradigms (Anderson, Bjork, \& Bjork, 1994; Bjork, 1972; Burwitz, 1974), it is assumed that retrieval practice or a forget instruction for a particular list of items induces a context change that modulates the retrieval of a subsequent list of items (notwithstanding that other processes might also contribute to these memory effects; e.g., Tempel, Aslan, \& Frings, 2016). Yet, offline contexts could also include external information-for example, in cued recall, if the cue is presented just before the participant has to retrieve a particular item.

Looking at retrieval processes discussed in the literature on action control, these also provide evidence for the influence of online and offline contexts. The influence of online contexts has, for instance, been shown in sequential prime-probe designs (e.g., Fox \& De Fockert, 1998; Wong, 2000), in which the involuntary retrieval of prime episodes influences probe performance if the prime and probe share several visual background features. In addition, task switching (for reviews of task switching, see, e.g., Kiesel et al., 2010; Koch, Gade, Schuch, \& Philipp, 2010) can be seen as a paradigm in action control in which online and offline contexts might influence retrieval. In particular, as an example for an online context, if one responds to a specific stimulus in task $\mathrm{A}$ and then has to respond to the same stimulus in task B (i.e., with another intention or task set), performance can be hampered by the involuntary retrieval of task A. However, changing the task set (e.g., presenting cues before the selection displays) and preventing stimulus repetition would be an instance of an offline context in task switching, since the cue presented before selection in trial $n$ modulates retrieval. In primeprobe designs it has been shown that temporal parameters modulate episodic retrieval (Neill, Valdes, Terry, \& Gorfein, 1992); that is, the time intervals between displays seem to provide an offline context in which prime retrieval was either hindered or furthered, depending on whether the prime episode was easily separable. Another example of an offline context was provided by Hommel, Memelink, Zmigrod, and Colzato (2014), who showed that the attentional set (i.e., a cue that signaled the task-relevant dimension) influenced the retrieval of bindings between relevant features and responses. Although the cue in itself could be seen as an external context, it was not presented together with stimulus selection; thus, it could be interpreted as an offline context that changed the attentional set with which participants responded.

We argue that these findings reflect a general mechanism that is observed in memory research as well as in action regulation-namely, the modulation of retrieval due to context changes: If there is no continuity of the encoding context (e.g., the task set) and the retrieval context, context shifts generally reduce, or even prevent, involuntary retrieval (although previous studies have shown that some effects can mostly survive a task switch; Pösse, Waszak, \& Hommel, 2006). Particularly interesting is the tentative evidence, based on the literature on task switching, that offline contexts can modulate retrieval in action control. However, changing the task set directly affects processing of the task-relevant features and responses. As a consequence, (1) the effects of task-set changes depend on the response and the target stimulus features (involuntary retrieval might thus always be accompanied by voluntary retrieval), and (2) by definition, task changes switch the task itself, so any modulation of retrieval might be masked by other effects, such as taskset inhibition (e.g., Kiesel et al., 2010).

Thus, we here used a different operationalization of offline context shifts - namely, changing the stimulus selection criterion while always preserving the task set. If the processing requirements are complex enough, then even without changing the task, a shift in the selection criterion should be enough to change the context in which information is processed, and thus should help reduce involuntary retrieval.

To test this assumption of a general influence of context shifts on involuntary retrieval, we used a distractor-todistractor priming paradigm. This paradigm taps involuntary retrieval because the distractor in trial $n$ retrieves the (target) response from trial $n-1$, leading to a distractor-based binding effect (e.g., Frings, Rothermund, \& Wentura, 2007; Frings, Schneider, \& Moeller, 2014; Mayr \& Buchner, 2006). This binding effect is reflected in the interaction of distractor relation and response relation, so that a repeated distractor retrieves the response from trial $n-1$, which would lead to facilitation on trial $n$ in the case of a response repetition, whereas it would lead to interference in the case of a response change (see Fig. 1).

The retrieval process is obviously involuntary, since participants always respond to the target and ignore the interfering distractor (which nevertheless triggers a retrieval process upon its repetition). The task remained the same during the entire experiment, yet the target selection criterion changed unpredictably from trial to trial. Specifically, participants had to discriminate target and distractor on the basis of a cue that indicated the relevant selection criterion (i.e., visual-spatial height or auditory pitch, depending on the target modality; see below). We defined cue-based changes of the selection criterion as representing a context shift, even though the task 
Trial $\mathrm{n}-1$

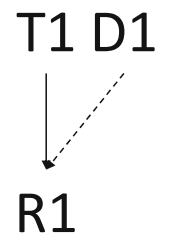

$T \sim$ target

$R \sim$ response to target

D distractor

Fig. 1 Scheme of the sequential distractor-to-distractor priming paradigm (see, e.g., Frings et al., 2007): Participants have to respond to the target while ignoring the distractor. The distractor D1 is bound to the response $\mathrm{R} 1$ in trial $n-1$ and upon repetition retrieves this response, which could be compatible or incompatible (as shown) with the response demanded on trial $n$. Note that the distractors and targets in the current trial are always incongruent and incompatible (although the effect also emerges if the distractor is not assigned to a response at all; Giesen et al., 2012)

itself remained the same throughout — actually, the cue can be seen as an offline context, since it was presented before the onset of the selection display. A similar "attention-switching" paradigm has been used in the auditory domain (Koch \& Lawo, 2014; Koch, Lawo, Fels, \& Vorländer, 2011), but in the present study we combined this paradigm with a distractor-to-distractor priming paradigm (Frings et al., 2007 ) to test the novel prediction that involuntary retrieval depends on continuity of the processing contexts.

We hypothesized that the context shift would weaken involuntary retrieval in stimulus selection criterion switch trials. In terms of an operational definition, the modulation of involuntary retrieval due to context shifts would be indicated by the following pattern: The Response Relation $\times$ Distractor Relation interaction taps the distractor-based binding effect, and we expected this binding effect to emerge in selection criterion repetition trials, whereas it should be diminished in selection criterion shift trials (i.e., we expected to find a threeway Selection Criterion Shift $\times$ Response Relation $\times$ Distractor Relation interaction).

To corroborate this argument and get independent replications, we varied the modality of the targets (visual vs. auditory) and the modality of the cue (visual vs. auditory) signaling the stimulus selection criterion shift in four independent groups. This design provided us with four independent replications of the predicted modulation of the distractor-based binding effect by context shifts, so we could examine the degree to which this modulation generalized across modality combinations of cues and targets.

\section{The experiment}

Participants responded to the identity of a target while ignoring a distractor in a sequential-priming task (i.e., each trial comprised a prime followed by a probe display to which participants responded). The distractor-based binding effect was reflected in the interaction of distractor and response repetitions. Before each prime and probe display, a cue indicated the selection criterion for the upcoming display (i.e., whether the target sound would have a high or a low pitch). We expected the distractor-based binding effect to depend on selection criterion repetitions.

\section{Method}

\section{Participants}

One hundred twenty-two students ( 85 female, 37 male) from the University of Trier took part in the experiment. One participant failed to report her age. The median age of the remaining sample was 22 years, with a range from 18 to 33 years. All participants took part in exchange for partial course credit.

The distractor-based binding effects were rather large to medium with respect to effect size in previous studies $(d z$ between 0.45 and $>1$ ). The four groups had a sample size of $N=30$ (only the group with auditory cues and auditory targets had $N=32$ participants). Given $N=30, \alpha=.05$, and an average effect of size $d z \geq 0.72$, the power to detect an effect of this size was $1-\beta=.98$ (G*Power 3.1.3; Faul, Erdfelder, Lang, \& Buchner, 2007).

\section{Materials}

The experiment was conducted using the E-Prime software (EPrime 2.0). Instructions and fixation marks as well as visual cues were shown in white on the black background of a standard CRT screen. In the auditory-target condition, the target and distractor stimuli were four digitized artificial sounds with a duration of $300 \mathrm{~ms}$ each. One of the sounds was similar to a school bell, another one resembled a release buzzer, a third one sounded like a beep, and the fourth sound was a siren-like wail. The Audacity software was used to convert the sounds into the high- and low-pitch stimuli. The high and low sounds were about an octave apart, and two different raters a priori judged the heights of the four different sounds as being subjectively the same. Consequently, each sound could be presented with either high or low pitch. In the visual-target condition, the targets and distractors were outlines of four different shapes (triangle, ellipsis, rectangle, and rhombus) that could be presented in light or dark blue. The shapes had a horizontal visual angle of $3.0^{\circ}$ to $4.1^{\circ}$ and a vertical visual angle of $2.1^{\circ}$ to $3.2^{\circ}$. In the auditorycue condition, a high $(1200-\mathrm{Hz})$ or a low $(100-\mathrm{Hz})$ pitched sound was presented for $100 \mathrm{~ms}$ before prime and probe onset. In the visual-cue condition, a white arrow pointing up or down, extending over a horizontal visual angle of $1.3^{\circ}$ and a vertical visual angle of $2.7^{\circ}$, was presented in the middle of the display, at a viewing distance of approximately $60 \mathrm{~cm}$. Participants responded via the $\mathrm{D}, \mathrm{F}, \mathrm{J}$, and $\mathrm{K}$ keys of a standard keyboard. 


\section{Procedure}

Participants were tested individually in soundproof chambers. Instructions were given on the screen and summarized by the experimenter. The conditions of the four experimental groups were made as structurally comparable as possible (i.e., same conceptual mapping of cue to selection criterion, arbitrary mappings of targets to responses). Participants were instructed to place the index and middle fingers of both hands on the $\mathrm{D}$, F, J, and K keys of the computer keyboard.

In the auditory-target condition, the bell sound corresponded to the D key, the buzzer to the F, the beep to the J, and the siren to the K. Participants always heard two different stimuli simultaneously at both ears - one at a high and one at a low pitch. In the visual-target condition, the triangle corresponded to the D key, the ellipsis to the F, the rectangle to the J, and the rhombus to the $\mathrm{K}$. The target and distractor shapes overlapped and were presented in the center of the screen.

A cue was presented for $100 \mathrm{~ms}$ before prime and probe stimuli onset, indicating the pitch/color in which the target would be presented in the following stimulus pair. In the auditory-cue condition, the cue was a high- or a low-pitched sound, and in the visual-cue condition, it was a white arrow on a black background pointing upward or downward. The highpitched cue sound and the cue arrow pointing up signaled that participants had to identify the shape presented in light blue or the high-pitched sound, respectively. The low-pitched cue sound and the cue arrow pointing down signaled that participants had to identify the shape presented in dark blue or the low-pitched sound, respectively. To identify the target, participants were instructed to press the corresponding key as quickly and accurately as possible.

Each trial featured a prime-probe sequence with the following events (see Fig. 2). Throughout each trial, a plus sign was presented as a fixation mark, with the exception of the cue displays in the visual-cue condition and the target and distractor presentation in the visual-target condition. Participants started each trial by pressing the space bar, and the prime cue was then presented for $100 \mathrm{~ms}$, followed by a silent period (blank display) lasting $400 \mathrm{~ms}$. Then the prime target and distractor stimuli were presented for $300 \mathrm{~ms}$. Participants pressed a key in response to the prime target. A silence followed that lasted for $500 \mathrm{~ms}$ until the probe cue was presented for $100 \mathrm{~ms}$, as before followed by a silent period lasting $400 \mathrm{~ms}$. Then the probe target and distractor stimuli were presented for $300 \mathrm{~ms}$, and participants pressed a key in response to the probe target. Finally, the fixation mark was replaced by an asterisk that indicated to the participant that the next trial could be started.

In response repetition trials, the target stimuli that were presented on the prime and probe displays were mapped to the same response, whereas on response change trials, the prime and probe target stimuli were mapped to different responses.
Distractor identity relation (repetition vs. change) was varied orthogonally to response relation, and selection criterion relation (cue repeated vs. changed) was varied orthogonally to both other factors, yielding eight experimental within-subjects conditions in total. In distractor repetition trials, the same sound/shape identity was presented as the distractor (i.e., in the noncued pitch/color) on the prime and probe displays, whereas in distractor change trials different sound/shape identities were presented as the prime and probe distractors. In selection criterion repetition trials, the prime and probe cues were identical (i.e., both high/arrow pointing up or both low/arrow pointing down), whereas different cues were presented before the prime and the probe stimulus pairs in selection criterion change trials (i.e., either prime cue high-pitched/pointing up and probe cue low-pitched/pointing down, or prime cue low-pitched/ pointing down and probe cue high-pitched/pointing up). For example, in an auditory-target trial with response change, distractor repetition, and selection criterion change, participants could be required to respond to a high-pitched bell sound while ignoring a low-pitched buzzer sound on the prime, and to respond to a low-pitched siren sound while ignoring a high-pitched buzzer sound on the probe. Note that further stimulus repetitions, such as target-to-distractor or distractor-to-target repetitions, were avoided.

Participants were randomly assigned to the four groups defined by target and cue modality (auditory targets and auditory cues, auditory targets and visual cues, visual targets and auditory cues, and visual targets and visual cues). Each participant worked through a single experimental block of 256 prime-probe sequences. The eight conditions defined by response relation, distractor relation, and selection criterion relation were each realized in 32 trials each. Target and distractor stimulus identities were randomly assigned to the prime and probe targets and distractors. Sound/shape identities were then changed to match the current trial type (e.g., if the trial included distractor repetition, the prime distractor identity was set to the probe distractor identity), and target and distractor pitch/color were adjusted according to the current trial type. Before the experimental block started, participants worked through two practice blocks in which they received feedback after each response. During the first practice block no distractors were presented, and participants practiced the stimulus identity-response mapping for 32 trials. The second practice block included 48 trials that were exactly like those in the experiment, with the exception that visual feedback was presented after each response.

\section{Design}

Response relation (repetition vs. change), distractor relation (repetition vs. change), and selection criterion relation (repeated vs. changed) were the within-subjects independent variables. In addition, cue modality (auditory vs. visual) and target modality (auditory vs. visual) were between-subjects 


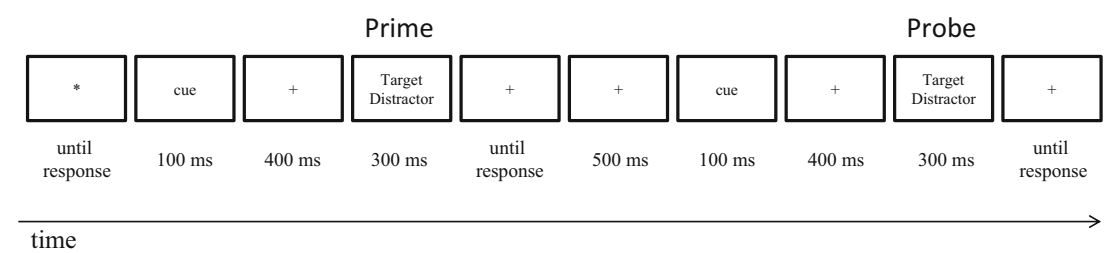

Fig. 2 Schematic display of the sequence of events in each trial. Note that cue modality and stimulus modality were fully varied (visual vs. auditory) between participants

variables; that is, we ran four different groups. Response time (RT) and error rate were the dependent variables.

\section{Results}

For the analysis of RTs, we considered only those trials with correct responses to both the prime and the probe (the error rates for the probe display were $41.1 \%$ in the auditory-target condition and $10.7 \%$ in the visual-target condition; the error rates for the prime displays were $46.6 \%$ in the auditory-target condition and $13.1 \%$ in the visual-target condition). RTs that were more than 1.5 interquartile ranges above the third quartile of the RT distribution of the auditory- or the visual-target condition (Tukey, 1977) and those shorter than $200 \mathrm{~ms}$ were excluded from the analysis. Due to these constraints, $68.1 \%$ of all auditory-target trials and $24.8 \%$ of all visual-target trials were discarded from the RT analyses. The high number of trials that had to be dropped from the RT analyses in the auditory-target groups led to fewer observations for the RT analysis, but note that the analysis of the error rates revealed exactly the same data pattern as the RTs of correct trials.

\section{RT analysis}

Our main analysis was a 2 (target modality: auditory vs. visual) $\times 2$ (cue modality: auditory vs. visual) $\times 2$ (selection criterion: repetition vs. change) $\times 2$ (response relation: repetition vs. change) $\times 2$ (distractor relation: repetition vs. change) mixed analysis of variance (ANOVA) on probe RTs (see Table 1 for the corresponding descriptive statistics for RTs and error rates).

Table 1 Mean response times (in milliseconds) and mean error rates (in percentages) as a function of target modality, cue modality, selection criterion relation, response relation, and distractor relation

\begin{tabular}{|c|c|c|c|c|c|c|}
\hline & \multicolumn{3}{|l|}{ Visual Targets } & \multicolumn{3}{|c|}{ Auditory Targets } \\
\hline & $\begin{array}{l}\text { Resp. } \\
\text { Repetition }\end{array}$ & $\begin{array}{l}\text { Resp. } \\
\text { Change }\end{array}$ & $\begin{array}{l}\text { Resp. } \\
\text { Repetition Effect }\end{array}$ & $\begin{array}{l}\text { Resp. } \\
\text { Repetition }\end{array}$ & $\begin{array}{l}\text { Resp. } \\
\text { Change }\end{array}$ & $\begin{array}{l}\text { Resp. } \\
\text { Repetition Effect }\end{array}$ \\
\hline \multicolumn{7}{|l|}{ Visual Cues } \\
\hline \multicolumn{7}{|l|}{ Selection Criterion Repetition } \\
\hline Distractor change & $824(5.1)$ & $950(12.2)$ & $126(7.1)$ & $1,454(32.1)$ & $1,729(42.1)$ & $275(10)$ \\
\hline Distractor repetition & $783(5.1)$ & $957(10.8)$ & $174(5.7)$ & $1,022(11.1)$ & $1,666(40.5)$ & $644(29.4)$ \\
\hline Distractor-based binding effect & $48(-1.4)$ & & & $369(19.4)$ & & \\
\hline \multicolumn{7}{|l|}{ Selection Criterion Change } \\
\hline Distractor change & $1,040(13.6)$ & $1,094(18.7)$ & $54(5.1)$ & $1,823(45.9)$ & $1,784(44.1)$ & $-39(-1.8)$ \\
\hline Distractor repetition & $1,040(13.5)$ & $1,041(16.7)$ & $1(3.2)$ & $1,724(43.7)$ & $1,728(40.4)$ & $4(-3.3)$ \\
\hline Distractor-based binding effect & $-53(-1.9)$ & & & $43(-1.5)$ & & \\
\hline \multicolumn{7}{|l|}{ Auditory Cues } \\
\hline \multicolumn{7}{|l|}{ Selection Criterion Repetition } \\
\hline Distractor change & $757(2.8)$ & $911(8.6)$ & $154(5.8)$ & $1,369(42.1)$ & $1,595(50.5)$ & $226(8.4)$ \\
\hline Distractor repetition & $713(1.6)$ & $901(6.8)$ & $188(5.2)$ & $1,064(14.0)$ & $1,520(44.8)$ & $456(30.8)$ \\
\hline Distractor-based binding effect & $34(-0.6)$ & & & $230(22.4)$ & & \\
\hline \multicolumn{7}{|l|}{ Selection Criterion Change } \\
\hline Distractor change & $1,059(15.6)$ & $1,075(16.0)$ & $16(0.4)$ & $1,675(56.7)$ & $1,553(45.7)$ & $-122(-11)$ \\
\hline Distractor repetition & $1,050(12.4)$ & $1,043(11.7)$ & $-7(-0.7)$ & $1,623(55.4)$ & $1,636(45.3)$ & $13(-10.1)$ \\
\hline Distractor-based binding effect & $-23(-1.1)$ & & & $109(0.9)$ & & \\
\hline
\end{tabular}

Response repetition effects (differences between response repetition and change trials averaged across distractor relation) and distractor-based binding effects (Response Relation $\times$ Distractor Relation interaction) are presented for each modality condition 
This analysis yielded significant main effects of selection criterion relation, $F(1,118)=319.88, p<.001, \eta_{\mathrm{p}}{ }^{2}=$ .73 ; response relation, $F(1,118)=102.07, p<.001, \eta_{\mathrm{p}}{ }^{2}=$ .46 ; and distractor relation, $F(1,118)=30.36, p<.001$, $\eta_{\mathrm{p}}{ }^{2}=.21$. RTs were shorter in selection criterion repetition trials $(M=1,113 \mathrm{~ms}, S D=353 \mathrm{~ms})$ than in selection criterion change trials $(M=1,369 \mathrm{~ms}, S D=411 \mathrm{~ms})$. RTs were also shorter in response repetition $(M=1,148$ $\mathrm{ms}, S D=339 \mathrm{~ms})$ than in response change $(M=1,319$ $\mathrm{ms}, S D=423 \mathrm{~ms}$ ) trials, and shorter if the distractor repeated $(M=1,186 \mathrm{~ms}, S D=337 \mathrm{~ms})$ than if the distractor identity changed $(M=1,280 \mathrm{~ms}, S D=427 \mathrm{~ms})$ from prime to probe. The main effect of target modality was also significant, $F(1,118)=175.46, p<.001, \eta_{\mathrm{p}}{ }^{2}=.60$, indicating faster responses to visual target stimuli $(M=$ $941 \mathrm{~ms}, S D=120 \mathrm{~ms})$ than to auditory target stimuli $(M=$ $1,507 \mathrm{~ms}, S D=312 \mathrm{~ms}$ ), whereas the main effect of cue modality was not significant, $F(1,118)=2.31, p=.131$, $\eta_{\mathrm{p}}^{2}=.02$.

Relevant for our hypotheses was the significant two-way interaction of response relation and distractor relation, $F(1$, $118)=16.12, p<.001, \eta_{\mathrm{p}}{ }^{2}=.12$, indicating the distractorbased binding effect between responses and distractor identities. Most interestingly, the three-way interaction of response relation, distractor relation, and selection criterion relation was significant as well, $F(1,118)=8.81, p=.004, \eta_{\mathrm{p}}{ }^{2}=.07$. Separate analyses revealed a significant distractor-based binding effect if the selection criterion was repeated, $F(1,121)=$
21.84, $p<.001, \eta_{\mathrm{p}}^{2}=.15$, but not if the selection criterion changed between prime and probe, $F<1$; that is, repeating a distractor triggered involuntary retrieval, but this was the case only if the selection criterion repeated.

The crucial three-way interaction was not further modulated by target modality, $F(1,118)=1.84, p=.178, \eta_{\mathrm{p}}{ }^{2}=.02$; cue modality, $F(1,118)=1.97, p=.163, \eta_{\mathrm{p}}{ }^{2}=.02$; or the interaction of target modality and cue modality, $F<1$ (see Fig. 3). All other effects were not directly relevant to testing our theoretical predictions, but for completeness they are reported in Table 2 of the Appendix.

\section{Error rate analysis}

The same ANOVA on error rates revealed the same pattern. The main effects of target modality, $F(1,118)=220.82, p$ $<.001, \eta_{\mathrm{p}}{ }^{2}=.65$; selection criterion relation, $F(1,118)=$ $119.84, p<.001, \eta_{\mathrm{p}}^{2}=.50$; response relation, $F(1,118)=$ $65.20, p<.001, \eta_{\mathrm{p}}{ }^{2}=.36$; and distractor relation, $F(1,118)$ $=52.74, p<.001, \eta_{\mathrm{p}}{ }^{2}=.31$, were all significant. Relevant to our hypotheses was the two-way interaction of response relation and distractor relation, $F(1,118)=12.04, p=.001$, $\eta_{\mathrm{p}}{ }^{2}=.09$, indicating also in the error rates the distractorbased binding effect between responses and distractor identities. The crucial three-way interaction of response relation, distractor relation, and selection criterion relation was significant, $F(1,118)=18.52, p<.001, \eta_{\mathrm{p}}^{2}=.14$, indicating a significant distractor-based binding effect only

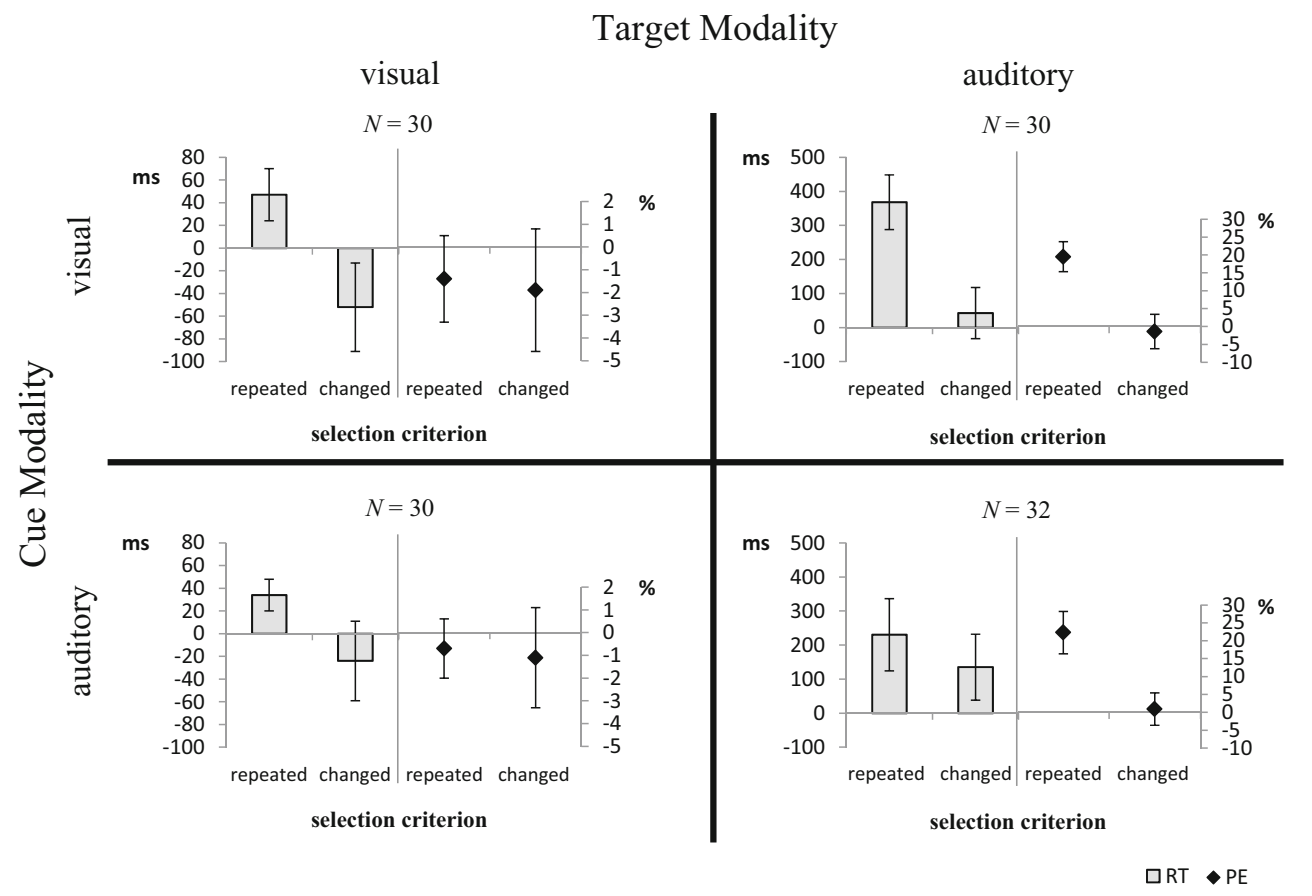

Fig. 3 Distractor-based binding effects (i.e., each data point reflects the interaction of response relation and distractor relation in that condition) as a function of target modality, cue modality, and selection criterion relation

for response times (RTs) and percentage errors (ER). Error bars depict standard errors of the means. Note the different scales for the dependent measures for the visual versus the auditory target modality 
with a repeated target selection criterion, $F(1,121)=$ $25.70, p<.001, \eta_{\mathrm{p}}{ }^{2}=.18$, but not with a changed selection criterion, $F(1,121)<2, p>.5, \eta_{\mathrm{p}}{ }^{2}<.01$. As for the RTs, this three-way interaction was not modulated by cue modality, $F<1$, or by an interaction of cue modality and target modality, $F<1$. However, it was modulated by target modality, $F(1,118)=17.11, p<.001, \eta_{\mathrm{p}}{ }^{2}=.13$, though the interaction was only of the ordinal type (i.e., the pattern was simply somewhat stronger for the auditory target modality). All other effects are reported in Table 2 of the Appendix.

\section{Discussion}

We set out to examine whether involuntary retrieval processes, which work at the interplay of selective attention and action control, crucially depend on the continuity of the processing context. To this end, we employed a distractor-to-distractor priming paradigm and combined it with trial-based variation of the stimulus selection criterion that needed to be applied for discriminating the target and distractor stimuli. Using this novel paradigm, we tested the prediction that involuntary distractor-based response retrieval is largely abolished if there is a change in the processing context, even if the task itself always remained the same.

Using this specific experimental paradigm combining the distractor-priming and criterion-switching methodologies, we found that a change in the offline context weakened involuntary distractor-based retrieval, as reflected in diminished distractor-based binding effects. In fact, if the selection criterion changed from prime to probe (even though the task remained the same), the otherwise robust distractor-based binding effect was no longer reliable. This pattern was found in four independent replications and across visual and auditory stimulus and cue modalities.

A closer look at the pattern of the interaction of response relation and distractor relation in the present data might lead to the impression that it is not exactly as predicted by the distractor-response retrieval model. The model predicts a distractor repetition advantage in response repetition trials and a disadvantage in response change trials. In the present data-as in many published studies on distractor-based response retrieval-the advantage during response repetition trials is evident, but the disadvantage predicted in the response change trials was not observed (instead, in some modality conditions a benefit was still observed). The reason is that in response change trials distractor-based retrieval (here leading to a cost effect) and a second, independent processdistractor inhibition (here leading to a beneficial effect) - can cancel each other out, leading to a nonsignificant net effect, or even a benefit in response change trials. Therefore, it has been argued that one must always interpret the Response Relation $\times$ Distractor Relation interaction and not solely interpret the response change condition, because the main effect of distractor inhibition might overshadow the retrieval effect (e.g., Frings \& Rothermund, 2011; Giesen, Frings, \& Rothermund, 2012; see Moeller, Rothermund, \& Frings, 2012, for exactly this pattern with auditory stimuli). Taken together, the data fit well with an explanation in terms of distractor-based retrieval of the previous prime responses (see also Mayr \& Bucher, 2006).

Note that we also found a clear effect of target modality. Performance was generally much worse when the selective-attention requirements referred to simultaneous auditory stimuli, as indicated by both clearly elevated RTs and increased error rates as compared to performance with visual targets. Yet, because both errors and RTs showed exactly the same theoretically predicted interaction pattern across target modalities and, more importantly, because the relevant three-way interaction was significant for both RTs and errors, and furthermore was modulated by cue modality for neither RTs nor errors, it is safe to conclude that, irrespective of the modality, involuntary retrieval in general is modulated by a change in the stimulus selection criterion.

Our data show a general principle of how the cognitive system modulates automatic behavior with a likewise automatic control mechanism. In particular, the involuntary retrieval of previous episodes (Logan, 1990) or event files (Hommel, 1998) elicited by stimulus, task, or response repetitions could lead to interference. Previous research has suggested that task changes or online context changes diminish retrieval (e.g., Wong, 2000); this may in fact be a typically helpful way to hedge against the retrieval of possibly incompatible episodes (because in most real-life situations, the responses changes when, for example, the context changes). However, context shifts need not be online to affect behavior. Here we showed that offline contexts can influence involuntary retrieval in (tasks that tap) action regulation. In fact, in many previous articles on context shifts in action control, the selection display that elicited retrieval was presented together with a different context (i.e., an online context), whereas here we used a cue presented before the selection display.

Although our results fit with recent results in action regulation (Hommel et al., 2014), they also can be interpreted through the lens of the instance theory of memory and attention (ITAM; Logan, 2002). In fact, in this enhancement of instance theory (Logan, 1988, 1990) retrieval is obligatory, in that it is driven by stimuli, but 
is also gated by such parameters as priorities and biases that constitute some kind of context. In particular, Schneider and Logan (2009) applied ITAM to task switching and assumed that the internal state formed by the representation of the task cue modulated what is obligatorily retrieved from the target, because the two formed a compound retrieval cue that jointly drives memory retrieval. Correspondingly, in terms of the ITAM, in our experiments the selection state biased obligatory prime retrieval.

Yet one might argue that it has remained unclear in the present set of experiments whether offline-context effects were due to the external cue that signaled the selection criterion for the upcoming display or to the internal selection criterion change itself. To investigate this issue, we replicated the condition with visual cues and visual stimuli in a control experiment $(N=30$; see the Appendix for details), with only the change that both cues (to the prime and probe) were always presented before the prime, and participants hence had to remember the selection criterion sequence for the upcoming prime-probe sequence. As a result, between probe retrieval and prime processing there was no change in external context, but on half the trials there was a change in the internal selection criterion. A significant distractor-response binding effect was observed for repeated selection criteria $(M=34 \mathrm{~ms}, S D=56 \mathrm{~ms}$; $t$ test against zero, $p=.002$ ), whereas no distractor-response binding effect was observed for changed selection criteria $(M=2 \mathrm{~ms}, S D=77 \mathrm{~ms} ; t$ test against zero, $p=.877)$. Yet the cues differed between selection repetition and selection change trials, so this control experiment cannot rule out the possibility that the cues still influenced retrieval. However, what we definitively can conclude from this control experiment is that external information does not need to be presented between prime encoding and probe retrieval to modulate retrieval in action control.

Finally, one might argue that the context shift was confounded with differences in the appearance of the distractor stimulus. The distractor stimuli appeared somewhat different in combination with context shifts versus nonshifts. In particular, for a visual condition, a change in the offline context would mean that the distracter stimulus could appear in a dark color in the prime trial but in a light color in the probe trial. Thus, the reduced influence of the distractor stimulus might reflect a perceptual mismatch between the prime and probe in the appearance of the distractor stimulus, rather than the impact of a context change. However, the literature suggests that perceptually similar distractors (Singh, Moeller, \& Frings, 2016), or even only conceptually similar distractor repetitions (Frings, Moeller, \& Rothermund, 2013), elicit distractor-based retrieval. Here, perceptually very similar distractor stimuli (dark vs. light bluish distractors of the same identity) elicited no retrieval in the context-shift conditions. In light of the background of the literature on S-R binding effects, this cannot easily be explained in terms of differences in stimulus appearance (Henson et al., 2014; Hommel, 1998), although differences in stimulus appearance might have boosted the impact of context shifts.

Overall, it is safe to conclude that a change in the offline context weakened retrieval of the prime episode. This is an effective way to modulate involuntary retrieval in situations in which this retrieval could lead to worse performance, even though the task itself remained the same. Just think of an everyday situation in which you do the same thing but with a different approachfor example, answering e-mails (private vs. business mails); it would probably not be appropriate to use the same salutation in both mails.

Our results have direct implications for the interpretation of several effects that are typically used to analyze action regulation and in which some kind of carryover effects are assumed, such as negative priming (see Frings, Schneider, \& Fox, 2015, for a recent review) or task switching (Kiesel et al., 2010; see also Koch, Frings, \& Schuch, 2017, for a recent discussion). For example, looking at the literature on negative priming, a variant of this task uses selection criterion repetitions versus shifts by default. In the location variant of this paradigm (e.g., Tipper, Brehaut, \& Driver, 1990), the target selection criterion changes from trial to trial. Interestingly, for visual location negative priming, this does not diminish the negative-priming effect (i.e., the repetition costs of repeating a prime distractor location as the probe target location), but it does destroy the otherwise robust attended repetition benefit (i.e., the repetition of the prime target location as the probe target location). Our results suggest that target repetition benefits in this paradigm seem to be based on involuntary retrieval of the previous prime episode (and thus are diminished by a shift in the stimulus selection criterion as a kind of offline context), whereas the location negative-priming effect seems to reflect another process (typically, distractor inhibition is assumed here) that is independent of involuntary retrieval. Thus, from this perspective, context shifts can be used to separate retrieval processes from other processes, such as response or distractor inhibition.

In conclusion, we presented evidence for a general mechanism that has long been accepted in memory research but is so far only selectively accepted as impacting upon action regulation. Changing the processing context modulates involuntary retrieval of previous $\mathrm{S}-\mathrm{R}$ episodes (even if the task remains the same) and, hence, shows how retrieval can be easily controlled. 


\section{Appendix}

Table $2 F$ values, $p$ values, and partial eta-squares for all effects of the 2 (target modality: auditory vs. visual) $\times 2$ (cue modality: auditory vs. visual) $\times 2$ (selection criterion: repetition vs. change) $\times 2$ (response relation: repetition vs. change) $\times 2$ (distractor relation: repetition vs. change) mixed ANOVA on $\mathrm{RTs}$; effects from the analogous ANOVA on error rates are depicted in parentheses

\begin{tabular}{|c|c|c|c|}
\hline Effect & $F$ & $p$ & $\eta_{\mathrm{p}}^{2}$ \\
\hline Target modality & $175.46(220.82)$ & $<.001(<.001)$ & $.60(.65)$ \\
\hline Cue modality & $2.31(1.13)$ & $.131(.290)$ & $.02(.01)$ \\
\hline Selection criterion & $319.88(119.84)$ & $<.001(<.001)$ & $.73(.50)$ \\
\hline Response relation & $102.07(65.20)$ & $<.001(<.001)$ & $.46(.36)$ \\
\hline Distractor relation & $30.36(52.74)$ & $<.001(<.001)$ & $.21(.31)$ \\
\hline Target modality $\times$ Cue modality & $0.85(5.30)$ & $.358(.023)$ & $.01(.04)$ \\
\hline Target modality $\times$ Selection criterion & $5.16(5.28)$ & $.025(.023)$ & $.04(.04)$ \\
\hline Target modality $\times$ Response relation & $12.24(4.00)$ & $.001(.048)$ & $.09(.03)$ \\
\hline Target modality $\times$ Distractor relation & $14.59(21.56)$ & $<.001(<.001)$ & $.11(.15)$ \\
\hline Cue modality $\times$ Selection criterion & $<0.001(0.43)$ & $.989(.515)$ & $<.001(.004)$ \\
\hline Cue modality $\times$ Response relation & $2.17(6.59)$ & $.143(.011)$ & $.02(.05)$ \\
\hline Cue modality $\times$ Distractor relation & $1.89(1.70)$ & $.172(.195)$ & $.02(.01)$ \\
\hline Selection criterion $\times$ Response relation & $127.21(139.01)$ & $<.001(<.001)$ & $.52(.54)$ \\
\hline Selection criterion $\times$ Distractor relation & $11.31(19.13)$ & $.001(<.001)$ & $.09(.14)$ \\
\hline Response relation $\times$ Distractor relation & $16.12(12.04)$ & $<.001(.001)$ & $.12(.09)$ \\
\hline Target modality $\times$ Cue modality $\times$ Selection criterion & $5.47(0.78)$ & $.021(.379)$ & $.04(.01)$ \\
\hline Target modality $\times$ Cue modality $\times$ Response relation & $2.08(0.30)$ & $.152(.583)$ & $.02(.003)$ \\
\hline Target modality $\times$ Cue modality $\times$ Distractor relation & $2.06(<0.001)$ & $.154(.985)$ & $.02(<.001)$ \\
\hline Target modality $\times$ Response relation $\times$ Distractor relation & $15.72(19.70)$ & $<.001(<.001)$ & $.12(.14)$ \\
\hline Cue modality $\times$ Selection criterion $\times$ Response relation & $0.13(4.78)$ & $.717(.031)$ & $.001(.04)$ \\
\hline Cue modality $\times$ Selection criterion $\times$ Distractor relation & $0.22(1.31)$ & $.640(.256)$ & $.002(.01)$ \\
\hline Cue modality $\times$ Response relation $\times$ Distractor relation & $0.02(0.40)$ & $.881(.526)$ & $<.001(.003)$ \\
\hline Selection criterion $\times$ Target modality $\times$ Response relation & $32.20(75.55)$ & $<.001(<.001)$ & $.21(.39)$ \\
\hline Selection criterion $\times$ Target modality $\times$ Distractor relation & $11.71(29.78)$ & $.001(<.001)$ & $.09(.20)$ \\
\hline Selection criterion $\times$ Response relation $\times$ Distractor relation & $8.81(18.52)$ & $.004(<.001)$ & $.07(.14)$ \\
\hline Target modality $\times$ Cue modality $\times$ Selection criterion $\times$ Response relation & $1.48(0.78)$ & $.227(.379)$ & $.01(.007)$ \\
\hline Target modality $\times$ Cue modality $\times$ Selection criterion $\times$ Distractor relation & $0.03(3.74)$ & $.854(.056)$ & $<.001(.03)$ \\
\hline Target modality $\times$ Cue modality $\times$ Response relation $\times$ Distractor relation & $0.10(0.11)$ & $.750(.738)$ & $.001(.001)$ \\
\hline Target modality $\times$ Selection criterion $\times$ Response relation $\times$ Distractor relation & $1.84(17.11)$ & $.178(<.001)$ & $.02(.13)$ \\
\hline Selection criterion $\times$ Cue modality $\times$ Response relation $\times$ Distractor relation & $1.97(0.002)$ & $.163(.962)$ & $.02(<.001)$ \\
\hline Target modality $\times$ Cue modality $\times$ Selection criterion $\times$ Response relation $\times$ Distractor relation & $0.96(0.01)$ & $.330(.947)$ & $.01(<.001)$ \\
\hline
\end{tabular}

Table 3 Mean reaction times (in milliseconds) and mean error rates (in percentages) of the control experiment with visual cues and visual stimuli as a function of selection criterion relation, response relation and distractor relation

\begin{tabular}{llr}
\hline & Response Repetition (RR) & Response Change (RC) \\
\hline $\begin{array}{l}\text { Selection criterion repetition } \\
\text { Distractor change }\end{array}$ & $644(2.1)$ & $905(6.7)$ \\
$\quad$ Distractor repetition & $590(0.9)$ & $919(7.9)$ \\
Selection criterion change & & $1,064(45.7)$ \\
$\quad$ Distractor change & $994(56.7)$ & $1,058(45.3)$ \\
Distractor repetition & $984(55.4)$ & \\
\hline
\end{tabular}


Table $4 F$ values, $p$ values, and partial eta-squares for all effects of the 2 (selection criterion: repetition vs. change) $\times 2$ (response relation: repetition vs. change) $\times 2$ (distractor relation: repetition vs. change) mixed ANOVA on RTs of the control experiment; effects from the analogous ANOVA on error rates are depicted in parentheses

\begin{tabular}{llll}
\hline Effect & $F$ & $p$ & $\eta_{\mathrm{p}}{ }^{2}$ \\
\hline Selection criterion & $246.87(20.17)$ & $<.001(<.001)$ & $<.001(<.001)$ \\
Response relation & $235.41(25.50)$ & $.061(.716)$ & $.90(.41)$ \\
Distractor relation & $3.80(0.14)$ & $<.001(.031)$ & $.89(.47)$ \\
Selection criterion $\times$ Response relation & $88.40(5.16)$ & $.499(.749)$ & $.03(.01)$ \\
Selection criterion $\times$ Distractor relation & $0.47(0.10)$ & $.030(.221)$ & $.02(.00)$ \\
Response relation $\times$ Distractor relation & $5.19(1.56)$ & $.099(.264)$ & $.15(.05)$ \\
Selection criterion $\times$ Target modality $\times$ Distractor relation & $2.91(1.30)$ & $.09(.04)$ \\
\hline
\end{tabular}

\section{References}

Anderson, M. C., Bjork, R. A., \& Bjork, E. L. (1994). Remembering can cause forgetting: Retrieval dynamics in long-term memory. Journal of Experimental Psychology: Learning, Memory, and Cognition, 20, 1063-1087. doi:10.1037/0278-7393.20.5.1063

Bjork, R. A. (1972). Theoretical implications of directed forgetting. In A. W. Melton \& E. Martin (Eds.), Coding processes in human memory (pp. 217-235). Washington, DC: Winston.

Burwitz, L. (1974). Proactive interference and directed forgetting in short-term motor memory. Journal of Experimental Psychology, 102,799-805.

Faul, F., Erdfelder, E., Lang, A.-G., \& Buchner, A. (2007). G*Power 3: A flexible statistical power analysis program for the social, behavioral, and biomedical sciences. Behavior Research Methods, 39, 175-191. doi:10.3758/BF03193146

Fox, E., \& De Fockert, J. W. (1998). Negative priming depends on primeprobe similarity: Evidence for episodic retrieval. Psychonomic Bulletin \& Review, 5, 107-113.

Frings, C., \& Rothermund, K. (2011). To be or not to be...included in an event file: Integration and retrieval of distractors in stimulus-response episodes is influenced by perceptual grouping. Journal of Experimental Psychology: Learning, Memory, \& Cognition, 37, 1209-1227.

Frings, C., Moeller, B., \& Rothermund, K. (2013). Retrieval of event files can be conceptually mediated. Attention, Perception, \& Psychophysics, 75, 700-709. doi:10.3758/s13414-013-0431-3

Frings, C., Rothermund, K., \& Wentura, D. (2007). Distractor repetitions retrieve previous responses to targets. Quarterly Journal of Experimental Psychology, 60, 1367-1377.

Frings, C., Schneider, K. K., \& Fox, E. (2015). The Negative Priming paradigm: An update and implications for selective attention. Psychonomic Bulletin \& Review, 22, 1577-1597. doi:10.3758/ s13423-015-0841-4

Frings, C., Schneider, K. K., \& Moeller, B. (2014). Auditory distractor processing in sequential selection tasks. Psychological Research, $78,411-422$.

Giesen, C., Frings, C., \& Rothermund, K. (2012). Differences in the strength of distractor inhibition do not affect distractor-response bindings. Memory \& Cognition, 40, 373-387. doi:10.3758/ s13421-011-0157-1

Henson, R. N., Eckstein, D., Waszak, F., Frings, C., \& Horner, A. J. (2014). Stimulus-response bindings in priming. Trends in Cognitive Sciences, 18, 376-384.

Hommel, B. (1998). Event files: Evidence for automatic integration of stimulus-response episodes. Visual Cognition, 5, 183-216. doi:10. $1080 / 713756773$
Hommel, B. (2004). Event files: Feature binding in and across perception and action. Trends in Cognitive Sciences, 8, 494-500. doi:10.1016/j. tics.2004.08.007

Hommel, B., Memelink, J., Zmigrod, S., \& Colzato, L. S. (2014). Attentional control of the creation and retrieval of stimulus-response bindings. Psychological Research, 78, 520-538.

Kenealy, P. M. (1997). Mood state-dependent retrieval: The effects of induced mood on memory reconsidered. Quarterly Journal of Experimental Psychology, 50A, 290-317.

Kiesel, A., Steinhauser, M., Wendt, M., Falkenstein, M., Jost, K., Philipp, A. M., \& Koch, I. (2010). Control and interference in task switching-A review. Psychological Bulletin, 136, 849-874. doi: 10.1037/a0019842

Koch, I., Frings, C., \& Schuch, S. (2017). Explaining response-repetition effects in task switching: Evidence from switching cue modality suggests episodic binding and response inhibition. Psychological Research. Advance online publication. doi:10.1007/s00426-0170847-9

Koch, I., Gade, M., Schuch, S., \& Philipp, A. M. (2010). The role of inhibition in task switching: A review. Psychonomic Bulletin \& Review, 17, 1-14. doi:10.3758/PBR.17.1.1

Koch, I., \& Lawo, V. (2014). Exploring temporal dissipation of attention settings in auditory task switching. Attention, Perception, \& Psychophysics, 76, 73-80. doi:10.3758/s13414-013-0571-5

Koch, I., Lawo, V., Fels, J., \& Vorländer, M. (2011). Switching in the cocktail party-Exploring intentional control of auditory selective attention. Journal of Experimental Psychology: Human Perception and Performance, 37, 1140-1147. doi:10.1037/a0022189

Logan, G. D. (1988). Toward an instance theory of automatization. Psychological Review, 95, 492-527. doi:10.1037/0033-295X.95.4. 492

Logan, G. D. (1990). Repetition priming and automaticity: Common underlying mechanisms? Cognitive Psychology, 22, 1-35. doi:10. 1016/0010-0285(90)90002-L

Logan, G. D. (2002). An instance theory of attention and memory. Psychological Review, 109, 376-400. doi:10.1037/0033-295X. 109.2.376

Mayr, S., \& Buchner, A. (2006). Evidence for episodic retrieval of inadequate prime responses in auditory negative priming. Journal of Experimental Psychology: Human Perception and Performance, 32, 932-943. doi:10.1037/0096-1523.32.4.932

Moeller, B., Rothermund, K., \& Frings, C. (2012). Integrating the irrelevant sound-Grouping modulates the integration of auditory distractors into event files. Experimental Psychology, 59, 258-264.

Moors, A., \& De Houwer, J. (2006). Automaticity: A theoretical and conceptual analysis. Psychological Bulletin, 132, 297-326. doi:10. 1037/0033-2909.132.2.297

Neill, W. T., Valdes, L. A., Terry, K. M., \& Gorfein, D. S. (1992). Persistence of negative priming: II. Evidence for episodic trace 
retrieval. Journal of Experimental Psychology: Learning, Memory, and Cognition, 18, 993-1000. doi:10.1037/0278-7393.18.5.993

Pösse, B., Waszak, F., \& Hommel, B. (2006). Do stimulus-response bindings survive a task switch? European Journal of Cognitive Psychology, 18, 640-651.

Roediger, H. L., III, \& McDermott, K. B. (1993). Implicit memory in normal human subjects. In F. Boller \& J. Grafman (Eds.), Handbook of neuropsychology (Vol. 8, 63-131). Amsterdam: Elsevier.

Schneider, D. W., \& Logan, G. D. (2009). Selecting a response in task switching: Testing a model of compound cue retrieval. Journal of Experimental Psychology: Learning, Memory, and Cognition, 35, 122-136. doi:10.1037/a0013744

Singh, T., Moeller, B., \& Frings, C. (2016). Five shades of grey: Generalization in distractor-based retrieval of S-R episodes. Attention, Perception, \& Psychophysics, 78, 2307-2312.

Tempel, T., Aslan, A., \& Frings, C. (2016). Competition dependence of retrieval-induced forgetting in motor memory. Memory \& Cognition, 44, 671-680. doi:10.3758/s13421-015-0578-3
Thomson, D. M., \& Tulving, E. (1970). Associative encoding and retrieval: Weak and strong cues. Journal of Experimental Psychology, 86, 255-262. doi:10.1037/h0029997

Tipper, S. P., Brehaut, J. C., \& Driver, J. (1990). Selection of moving and static objects for the control of spatially directed action. Journal of Experimental Psychology: Human Perception and Performance, 16, 492-504. doi:10.1037/0096-1523.16.3.492

Tukey, J. (1977). Exploratory data analysis. Reading: AddisonWesley

Ucros, C. G. (1989). Mood state-dependent memory: A meta-analysis. Cognition and Emotion, 3, 139-169.

Wong, K. F. E. (2000). Dissociative prime-probe contextual similarity effects on negative priming and repetition priming: A challenge to episodic retrieval as a unified account on negative priming. Journal of Experimental Psychology: Learning, Memory, and Cognition, 26, $1411-1422$. 